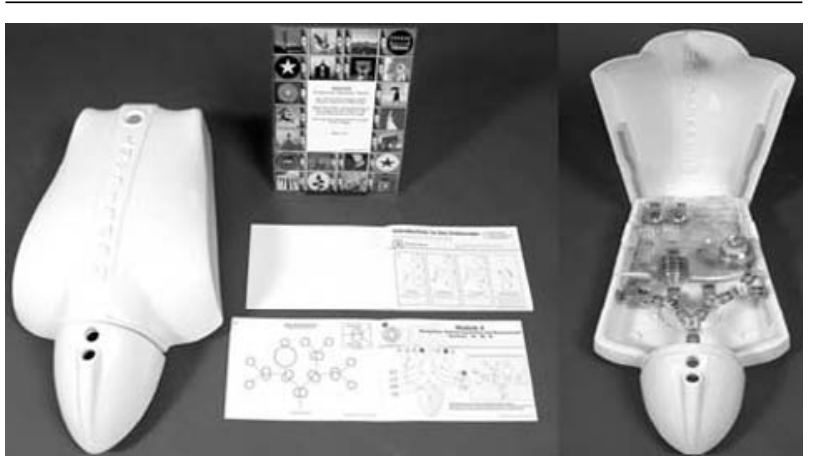

FIGURE Dexter® Endoscopic Dexterity Trainer.

\section{Modification of the Lipp maneuver for blind insertion of the Esophageal- Tracheal Combitube}

To the Editor:

Modifications of the original technique have been suggested to facilitate the insertion technique of the Esophageal-Tracheal Combitube ${ }^{\circledR}$ (ETC; TycoHealthcare, Pleasanton, CA, USA). ${ }^{1-3}$ We wish to communicate a modification of the "so-called Lipp maneuver", which is used to facilitate blind insertion of the device. Originally, Dr. Markus Lipp recommended bending the ETC in the portion between the balloons (Figure) for a few seconds in order to augment the preformed curvature, allowing an even more rapid placement.

It also became apparent that it is very important to avoid contact of the tip of the ETC with the posterior pharyngeal wall during insertion, since the posterior pharyngeal wall is very friable and bleeds easily. Bloody stripes are often seen after the use of the laryngeal mask airway, laryngeal tube, etc., because all these devices share the same mechanism of insertion, which is its application against the palate and the posterior pharyngeal wall.

Contrary to upper airway devices, insertion of the ETC does not include slipping it against the palate and posterior pharyngeal wall. The tip of the ETC should be applied against the tongue, thus avoiding damage to the pharynx.

In order to improve the efficacy and safety of the blind insertion of the ETC, we strongly recommend keeping the ETC bent as long as possible prior to insertion in the oropharyngeal cavity. To achieve this, the ETC should be held as shown in the Figure.
Maintaining the ETC bent, together with a rapid insertion into the oropharyngeal cavity will enable the tip to reach the esophagus before the ETC recovers its original, less curved shape, making insertion fast and safe.

Of course, during laryngoscopic insertion it is not necessary to perform this maneuver because the ETC is inserted under direct vision while avoiding the posterior pharyngeal wall.

Ricardo M. Urtubia MD*

Michael Frass MD $\dagger$

Thomas Staudinger MD $\dagger$

Peter Krafft MD $\dagger$

Santiago, Chile*

Vienna, Austria†

\section{References}

1 Frass $M$, Standinger TH, Losert H, Krafft P. Airway management during cardiopulmonary resuscitation--a comparative study of bag-valve-mask, laryngeal mask
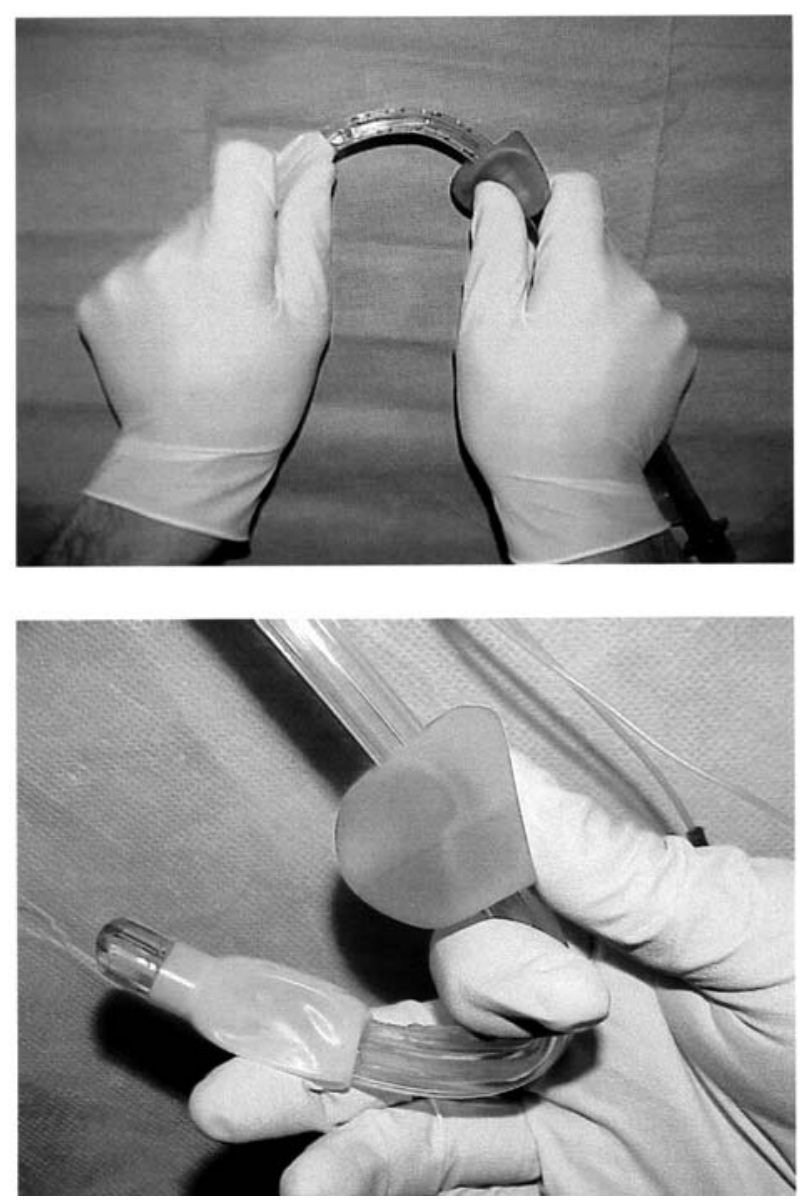

$\overline{\text { FIGURE Original (above) and modified (below) Lipp maneuver. }}$ 
airway and Combitube in a bench model (Letter).

Resuscitation 1999; 43: 80-1.

2 Urtubia RM, Frass M, Agrò F. New insertion technique for the Esophageal-Tracheal Combitube ${ }^{\circledR}$ (Letter). Acta Anaesthesiol Scand 2002; 46: 340-1.

3 Urtubia RM. 'Tricks of the trade' with the Esophageal-Tracheal Combitube (Letter). Acta Anaesthesiol Scand 2002; 46: 340. 